\title{
Socioeconomic and sex differences in health care utilisation, counselling on cardiovascular disease (CVD) risk factors, and CVD risk factors control in the Polish population. The WOBASZ II Study
}

\author{
Magdalena Kozela ${ }^{1}$, Maciej Polak ${ }^{1}$, Agnieszka Doryńska ${ }^{1}$, Agnieszka Borowiec ${ }^{2}$, Wojciech Bielecki ${ }^{3}$, \\ Krystyna Kozakiewicz ${ }^{4}$, Andrzej Tykarski ${ }^{5}$, Tomasz Zdrojewski ${ }^{6}$, Wojciech Drygas ${ }^{2,3}$, Andrzej Pająk ${ }^{1}$ \\ 'Chair of Epidemiology and Population Studies, Institute of Public Health, Jagiellonian University Medical College, Krakow, Poland \\ 2Department of Epidemiology, Cardiovascular Disease Prevention, and Health Promotion, Institute of Cardiology, Warsaw, Poland \\ ${ }^{3}$ Department of Social and Preventive Medicine, Medical University of Lodz, Lodz, Poland \\ ${ }^{4} 3^{\text {rd }}$ Department of Cardiology, Medical University of Silesia, Katowice, Poland \\ ${ }^{5}$ Department of Hypertension, Angiology, and Internal Medicine, Poznan University of Medical Sciences, Poznan, Poland \\ ${ }^{6}$ Department of Arterial Hypertension and Diabetology, Medical University of Gdansk, Gdansk, Poland
}

\begin{abstract}
Background: Socioeconomic status (SES) is one of the causes of inequality in health care utilisation. There is no information whether differences in SES influence the frequency of counselling on cardiovascular disease (CVD) risk factors or risk factors control.

Aim: We sought to assess the relationship between SES and the frequency of medical consultations, hospitalisations, counselling on CVD risk factors, and successful CVD risk factors control.

Methods: WOBASZ II was a cross-sectional study targeting a representative sample of the Polish population. Trained nurses interviewed participants using a standard questionnaire, collecting information on education, income, self-rated health, and health care utilisation. Blood samples were collected according to standardised methods.

Results: A total of 2303 men and 2848 women were included in the analysis. Compared to those with low SES, men with medium or high SES were $68 \%$ and $46 \%$ more likely to use medical consultations, respectively. Women with medium and high SES used medical consultations $60 \%$ more often than those with low SES. Men with medium and high SES had blood pressure measured more often (by $31 \%$ and $43 \%$, respectively), and more frequently received nutritional (by $45 \%$ and $59 \%$, respectively) and physical activity counselling (by $92 \%$ and $122 \%$, respectively). No differences in CVD risk factors control were found.

Conclusions: High SES was associated with more frequent medical visits in both sexes. The associations of SES with counselling on CVD risk factors substantially differed between the sexes in favour of men with high SES. However, more complex consultations in high SES men were not followed by better CVD risk factors control.
\end{abstract}

Key words: cardiovascular disease prevention, health care utilisation, socioeconomic status

Kardiol Pol 2018; 76, 11: 1516-1524

\section{INTRODUCTION}

Cardiovascular disease (CVD), the main cause of deaths in Poland, and particularly coronary heart disease, used to be commonly regarded in Poland as a "disease of managers." It was documented, however, that it affects people with lower education level much more. Nowadays, the social gradient in CVD is well described, indicating higher CVD rates and higher prevalence of CVD risk factors in groups with lower socioeconomic status (SES) [1-3]. In Poland, similarly to other European countries, a strong social gradient in CVD mortality was ob-

\section{Address for correspondence:}

Dr. Magdalena Kozela, Chair of Epidemiology and Population Studies, Jagiellonian University Medical College, ul. Grzegórzecka 20, $31-531$ Kraków, Poland, tel: +48 1243328 38, e-mail: m.kozela@uj.edu.pl 
served. Estimates of the Polish Government Population Council (Rządowa Rada Ludnościowa) not only show that groups with low education have higher CVD mortality rates, but also that the differences in mortality are increasing over time [4]. Two recent publications by Podolecka et al. [5] and Nadrowski et al. [6] confirmed strong negative relationships between SES and the prevalence of CVD risk factors as well as between SES and CVD risk measured as SCORE index in the Polish population. Higher education level was associated with a lower prevalence of hypertension, smoking, overweight, obesity, diabetes, and dyslipidaemia. Higher SCORE risk was found in unprivileged groups, compared to persons with high SES.

The causes of the observed health disparities are not fully understood. One of the possible reasons is better access to health care in persons with high SES. In Poland the level of expenditure on health care in relation to gross domestic product is among the lowest in Europe. It is estimated that private expenditure constitutes up to $23 \%$ of all costs of the health care sector $[7,8]$. As a consequence, it is likely that access to health care depends on the possibility of incurring health expenses.

On the other hand, SES inequalities in CVD prevalence are well known and described in populations of Western European countries, where the share of public financing of health care is greater than in Poland [1-3], and the possibility of bearing private expenditure for health care may have a lower impact. If so, the interpretation that persons with lower SES develop CVD more often because they are unable to pay for the required health care services could be false.

The negative relationship between SES and CVD prevalence might also be the result of higher health literacy in people with high levels of education. The Health Literacy Survey study proved that in Poland the relationship between education level and health literacy is one of the highest in Europe. Higher health competences can lead to better use of the health care system, and the ability to search, understand, evaluate, and apply information. It was also found that health competences were positively associated with self-rated health status $[9,10]$. The relationship between health competences and the frequency of doctor visits is still unclear, and the question of the quality of health care provided for patients with different SES in Poland remains open.

The objective of the present study was to assess the relationship between SES and the frequency of medical consultations, hospitalisations, counselling on CVD risk factors, and successful CVD risk factors control.

\section{METHODS}

\section{Study population}

The Multi-centre National Population Health Examination Survey (WOBASZ II study) was a nationwide, cross-sectional study conducted in Poland between 2013 and 2014. A detailed description of the main goals, methods, and sample selection of the study was published previously [11]. A summary of the information relevant for this paper is given below. The sample was recruited from the national population register, using a multistage sampling design. Recruitment in WOBASZ II aimed to achieve a sample representative of the total Polish population aged 20 years and older. Out of 15,200 randomly selected residents, 1557 were not eligible for the study (deceased, moved away, or unreachable due to poor health status). The final response rate was $45.5 \%$. All the WOBASZ II participants gave written consent to take part in the study.

\section{Data collection}

Trained nurses interviewed the participants according to a standard questionnaire, which gathered information on education, income, self-rated health, and health care utilisation characteristics. Blood collection for biochemical tests was performed following standardised methods. Information obtained from the questionnaire, physical examination, and biochemical blood test enabled the assessment of CVD risk factors control.

\section{Measurements}

Socioeconomic status was defined according to the method using the algorithm developed by Kozakiewicz et al. [12], based on the ATTICA Study experience $[12,13]$. SES score was calculated by multiplying ordinal numerical values assigned to consecutive categories of education level and family income per capita (education: lack of formal education or primary or middle school $=1$, vocational school based on primary or middle school $=2$, secondary $=3$, post-secondary or bachelor degree $=4$, university $=5$; income in PLN: $<500=1$, $501-1000=2,1001-1500=3,1501-2000=4,2001-$ $-2500=5,2501-3000=6,>3000=7$ ); answers "I do not know" and "I refuse to disclose" were excluded. The SES score ranged from 1 to 35. In further analysis participants were divided into three subgroups of low, medium, and high SES according to tertile values of SES score distribution (Table 1). Figure 1 shows the distribution of SES scores and cut-off values.

Health care utilisation was assessed through the question about hospitalisation and/or medical consultation in the previous 12 months (yes/no). Counselling on CVD risk factors was assessed using the following questions: During a typical medical visit, do you usually: 1) Have your blood pressure (BP) measured? 2) Receive advice regarding smoking cessation? 3) Receive nutritional advice? 4) Receive advice about increasing physical activity?

Blood pressure was measured using an automatic UA-631 device, after five minutes' rest, on the right arm, in the sitting position, three times at 1-min intervals. In the BP analysis, the average from the second and third measurements was used. Blood samples were collected and centrifuged at regional centres where they were frozen at $-20^{\circ} \mathrm{C}$. Then, serum samples were transported in dry ice to a central laboratory in Warsaw. Analysis of lipid fraction and glucose concentrations was performed using an automated enzymatic method. 
Table 1. Distribution of education, income, and the derived socioeconomic status (SES) score

\begin{tabular}{|lccc|}
\hline & Total & Men & Women \\
\hline Education $(n=6162):$ & & & \\
Lack of formal education, primary, or middle school & $1053(17.1)$ & $408(14.8)$ & $645(18.9)$ \\
Vocational based on primary or on middle school & $1473(23.9)$ & $856(31.1)$ & $617(18.1)$ \\
Secondary & $1921(31.2)$ & $904(32.8)$ & $1017(19.9)$ \\
Post-secondary or bachelor degree & $484(7.9)$ & $117(4.2)$ & $367(10.8)$ \\
University & $1231(20.0)$ & $472(17.1)$ & $759(22.3)$ \\
Family income per capita $(n=6145):$ & & & \\
$<500$ PLN & $633(10.3)$ & $267(9.7)$ & $366(10.8)$ \\
501-1000 PLN & $1690(27.5)$ & $696(25.4)$ & $994(29.2)$ \\
1001-1500 PLN & $1335(21.7)$ & $554(20.2)$ & $781(23.0)$ \\
1501-2000 PLN & $766(12.5)$ & $380(13.8)$ & $386(11.4)$ \\
2001-2500 PLN & $346(5.6)$ & $180(6.6)$ & $166(4.9)$ \\
$2501-3000$ PLN & $181(3.0)$ & $100(3.6)$ & $81(2.4)$ \\
$>3000$ PLN & $206(3.4)$ & $128(4.7)$ & $78(2.3)$ \\
I do not know & $413(6.7)$ & $179(6.5)$ & $234(6.9)$ \\
I refuse to disclose & $575(9.4)$ & $262(9.5)$ & $313(9.2)$ \\
SES score (n = 5151) & $6(4-12)$ & $6(4-12)$ & $6(4-12)$ \\
\hline
\end{tabular}

Data are shown as number (percentage) or median (interquartile range)

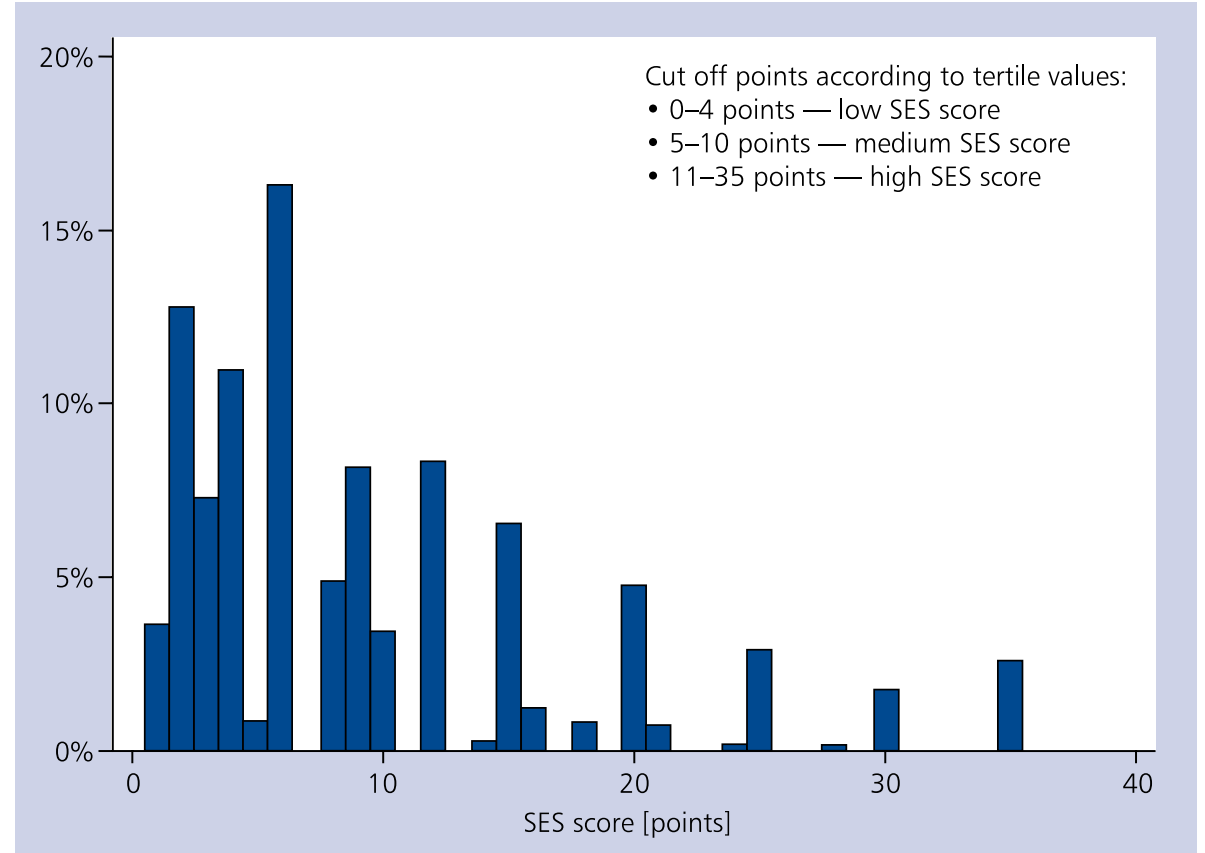

Figure 1. Distribution of the socioeconomic status (SES) score

Control of CVD risk factors was determined in persons in whom they were identified by the doctor. Treatment targets were defined according to the European Guidelines on CVD prevention in clinical practice, as applied for the earlier WOBASZ publications [14, 15]. Successful control of hypertension was defined as systolic BP $<140 \mathrm{mmHg}$ and diastolic BP $<90 \mathrm{mmHg}$. Controlled diabetes in patients with a clinical diagnosis was defined as fasting glucose $<6 \mathrm{mmol} / \mathrm{L}$. 


\section{Statistical analysis}

Distributions of continuous variables were presented for the total sample and for SES subgroups as mean and standard deviation in the case of normal distribution. Otherwise, median and interquartile range was given. Differences in distributions of the continuous variables according to SES categories were assessed using ANOVA or Kruskal-Wallis test, respectively. Categorical variables were presented as raw numbers and percentages. Comparison of distributions of categorical variables according to SES categories was done using $\chi^{2}$ test. The main statistical method was multivariable logistic regression. Due to the strong confounding effect of sex and self-rated health, the analysis was run separately for men and women using the following models: 1 ) adjusted for age only, 2) adjusted for age and self-rated health, and 3) adjusted for age self-rated health, marital status, and community size. The results were presented as odds ratios with $95 \%$ confidence intervals. All analyses were done using statistical package STATA v. 14 (Stata Corp. LP, College Station, TX, USA). Statistical significance was accepted at the level of $p<0.05$.

\section{RESULTS}

Out of the 2760 men and 3410 women who participated in the WOBASZ II study, 455 men and 474 women were excluded due to missing data on SES (Table 1). A total of 2303 men and 2848 women were included in the present analysis. Table 2 presents descriptive statistics of age, sex, size of the community, self-rated health, health care utilisation, and CVD risk factors control for the total sample and according to SES, for men and women separately. Mean age was $48.8 \pm 16.32$ years in men and $50.2 \pm 16.48$ years in women, and negative correlations between age and SES category were found ( $p<0.001$ ). All participants were equally distributed between small, medium, and large communities, although a substantially higher representation of high SES was found in large communities as compared to small ones in both sexes $(p<0.001)$. The vast majority of men and women declared their health status as good or very good, and a strong positive association with SES category was observed ( $p<0.001$ ). Over $70 \%$ of men and nearly $84 \%$ of women had at least one medical consultation within 12 months before the examination (most participants consulted a general practitioner [GP] and only $8 \%$ of them consulted a specialist), and about $13 \%$ of both male and female participants were hospitalised. During a typical medical visit over $60 \%$ of participants declared to have had their BP measured. Counselling on nutrition and physical activity was provided for nearly $31 \%$ and $26 \%$ of men and $29 \%$ and $22 \%$ of women, respectively. Anti-tobacco advice was given to about a half of the smokers. The highest percentages of BP measurements were found in participants with low SES ( $p=0.008$ in men and $p<0.001$ in women). In men, counselling on nutrition and smoking cessation was the most frequent in participants from the middle SES group. The percentage of participants who reached treatment targets for any of the CVD risk factors was rather low. In men BP was successfully controlled in $43 \%$ of participants, whereas diabetes and hypercholesterolaemia were successfully controlled in $27 \%$ and $18 \%$ of participants, respectively. In women, hypertension was controlled in nearly $50 \%$ of participants. As many as $26 \%$ and $20 \%$ of women were at treatment targets in the case of diabetes and hypercholesterolaemia, respectively. Risk factors control did not differ according to the SES category, with the exception of hypertension control in women, which was positively related with SES $(p=0.029)$.

The association between SES and health care utilisation and CVD risk factors control, according to sex, at different levels of standardisation is presented in Table 3. Age-adjusted odds ratios show a negative association between SES and hospitalisations and positive associations between SES and medical consultations, nutritional counselling, and physical activity counselling in men. In women, negative associations were found between SES and hospitalisations and BP measurement, while a positive correlation with medical consultations was found. The inclusion of self-rated health waived the association between SES and hospitalisations in both sexes and with BP measurements in women. Finally, in a fully adjusted model, an increase in medical consultations by SES category was observed in both sexes. Compared to those with low SES, men with medium or high SES were more likely to use medical consultations, by $68 \%$ and $46 \%$, respectively. Women with medium and high SES used medical consultations $60 \%$ more often than women with low SES. It was the only significant association between SES and health care utilisation characteristics that was observed in women. In contrast, men with moderate and high SES more often had their BP measured (by $31 \%$ and $43 \%$, respectively), and more frequently received nutritional counselling (by $45 \%$ and $59 \%$, respectively) and physical activity counselling (by $92 \%$ and more than twofold, respectively). No significant differences in hypertension, diabetes, or hypercholesterolaemia control were found in men or in women.

\section{DISCUSSION}

High SES was associated with more frequent medical visits in both sexes. The associations with counselling on CVD risk factors differed substantially between the sexes in favour of men with high SES. No statistically significant differences were found in the frequency of hospitalisations or control of main CVD risk factors according to SES category.

More frequent use of medical consultations in participants with higher SES is not surprising and can be explained by the fact that these persons could have better access to additional outpatient care (outside the public system) due to private health insurance or the possibility to afford extra consultations. According to the Central Statistical Office of Poland the proportion of people who have private health insurance does not exceed $10 \%$, and it is strongly related to income per capita. In 2013 over $60 \%$ of private health insurance 
Table 2. Distribution of age, sex, size of the community, health care utilisation, counselling on cardiovascular disease (CVD) risk factors, and CVD risk factors control by socioeconomic status (SES) category

\begin{tabular}{|c|c|c|c|c|c|}
\hline & & & SES & & \\
\hline & Total & Low & Medium & High & $\mathbf{P}$ \\
\hline Men & & & & & \\
\hline Age $(n=2760)$ & $48.8 \pm 16.32$ & $54.5 \pm 16.17$ & $49.62 \pm 15.75$ & $44.27 \pm 15.34$ & $<0.001$ \\
\hline Marital status $(n=2303)$ : & & & & & 0.111 \\
\hline Married or cohabiting & $1621(70.39)$ & $577(71.32)$ & $561(72.11)$ & $483(67.46)$ & \\
\hline Widowed/separated & $682(29.61)$ & $232(28.68)$ & $217(27.89)$ & $233(32.54)$ & \\
\hline Community $(n=2303)$ : & & & & & $<0.001$ \\
\hline Small & $798(34.65)$ & $434(53.65)$ & $233(29.95)$ & $131(18.30)$ & \\
\hline Medium & $691(30.00)$ & $247(30.53)$ & $259(33.29)$ & $185(25.84)$ & \\
\hline Large & $814(35.35)$ & $128(15.82)$ & $286(36.76)$ & $400(55.87)$ & \\
\hline Medical consultation in the last 12 months $(n=2301)$ & $1647(71.58)$ & $569(70.42)$ & $585(75.29)$ & $493(68.85)$ & 0.015 \\
\hline Hospitalisation in the last 12 months $(n=2301)$ & $299(12.99)$ & $128(15.84)$ & $106(13.64)$ & $65(9.08)$ & $<0.001$ \\
\hline Blood pressure measurement $(n=2094)$ & $1291(61.65)$ & $470(64.83)$ & $448(62.75)$ & $373(56.95)$ & 0.008 \\
\hline Anti-tobacco advice $(\mathrm{n}=718)$ & $404(56.27)$ & $162(59.34)$ & $150(60.24)$ & $92(46.94)$ & 0.008 \\
\hline Nutritional advice $(n=2073)$ & $645(31.11)$ & $218(30.32)$ & $234(33.33)$ & $193(29.6)$ & 0.284 \\
\hline Physical activity advice $(n=2068)$ & $546(26.40)$ & $151(20.94)$ & $206(29.68)$ & $189(28.94)$ & $<0.001$ \\
\hline Hypertension control $(n=819)$ & $352(42.98)$ & $116(38.41)$ & $120(44.12)$ & $116(47.35)$ & 0.099 \\
\hline Diabetes control $(n=192)$ & $51(26.56)$ & $22(25.88)$ & $18(26.47)$ & $11(28.21)$ & 0.963 \\
\hline Hypercholesterolaemia control $(n=660)$ & $120(18.18)$ & $36(19.35)$ & $49(20.08)$ & $35(15.22)$ & 0.346 \\
\hline Self-rated health $(n=2253)$ : & & & & & $<0.001$ \\
\hline Good/very good & $1542(68.44)$ & $422(53.69)$ & $518(68.25)$ & $602(85.03)$ & \\
\hline Moderate & $614(27.25)$ & $303(38.55)$ & $211(27.8)$ & $100(14.12)$ & \\
\hline Poor/very poor & $97(4.31)$ & $61(7.76)$ & $30(3.95)$ & $6(0.85)$ & \\
\hline Women & & & & & $<0.001$ \\
\hline Age $(n=3409)$ & $50.2 \pm 16.48$ & $58.25 \pm 16.56$ & $48.09 \pm 15.36$ & $45.34 \pm 14.46$ & \\
\hline Marital status $(n=2303)$ : & & & & & 0.002 \\
\hline Married or cohabiting & $1041(36.55)$ & $405(40.83)$ & $324(33.26)$ & $312(35.37)$ & \\
\hline Widowed/separated & $1807(63.45)$ & $587(59.17)$ & $650(66.74)$ & $570(64.63)$ & \\
\hline Community $(n=2848)$ : & & & & & $<0.001$ \\
\hline Small & $1033(36.27)$ & $530(53.43)$ & $325(33.37)$ & $178(20.18)$ & \\
\hline Medium & $863(30.30)$ & $306(30.85)$ & $304(31.21)$ & $253(28.68)$ & \\
\hline Big & $952(33.43)$ & $156(15.73)$ & $345(35.42)$ & $451(51.13)$ & \\
\hline Medical consultation in the last 12 months $(n=2845)$ & $2386(83.87)$ & $832(83.87)$ & $819(84.35)$ & $735(83.33)$ & 0.390 \\
\hline Hospitalisation in the last 12 months $(n=2848)$ & $390(13.69)$ & $178(17.94)$ & $116(11.91)$ & $96(10.88)$ & $<0.001$ \\
\hline Blood pressure measurement $(n=2711)$ & $1649(60.83)$ & $672(71.87)$ & $533(57.56)$ & $444(52.24)$ & $<0.001$ \\
\hline Anti-tobacco advice $(\mathrm{n}=615)$ & $319(51.87)$ & $109(56.19)$ & $119(49.38)$ & $91(50.56)$ & 0.338 \\
\hline Nutritional advice $(\mathrm{n}=2659)$ & $768(28.88)$ & $330(36.63)$ & $233(25.35)$ & $205(24.43)$ & $<0.001$ \\
\hline Physical activity advice $(n=2647)$ & $574(21.68)$ & $223(25.06)$ & $179(19.44)$ & $172(20.57)$ & 0.010 \\
\hline Hypertension control $(n=1040)$ & $513(49.33)$ & $219(45.15)$ & $164(51.41)$ & $130(55.08)$ & 0.029 \\
\hline Diabetes control $(n=232)$ & $62(26.72)$ & $42(31.58)$ & $13(21.67)$ & $7(17.95)$ & 0.141 \\
\hline Hypercholesterolaemia control $(n=889)$ & $178(20.02)$ & $70(22.01)$ & $56(18.30)$ & $52(19.62)$ & 0.502 \\
\hline Self-rated health $(n=2804)$ : & & & & & $<0.001$ \\
\hline Good/very good & $1801(64.23)$ & $454(46.71)$ & $674(70.28)$ & $673(77.09)$ & \\
\hline Moderate & $856(30.53)$ & $420(43.21)$ & $250(26.07)$ & $186(21.31)$ & \\
\hline Poor/very poor & $147(5.24)$ & $98(10.08)$ & $35(3.65)$ & $14(1.60)$ & \\
\hline
\end{tabular}

Data are shown as mean \pm standard deviation or number (percentage). 


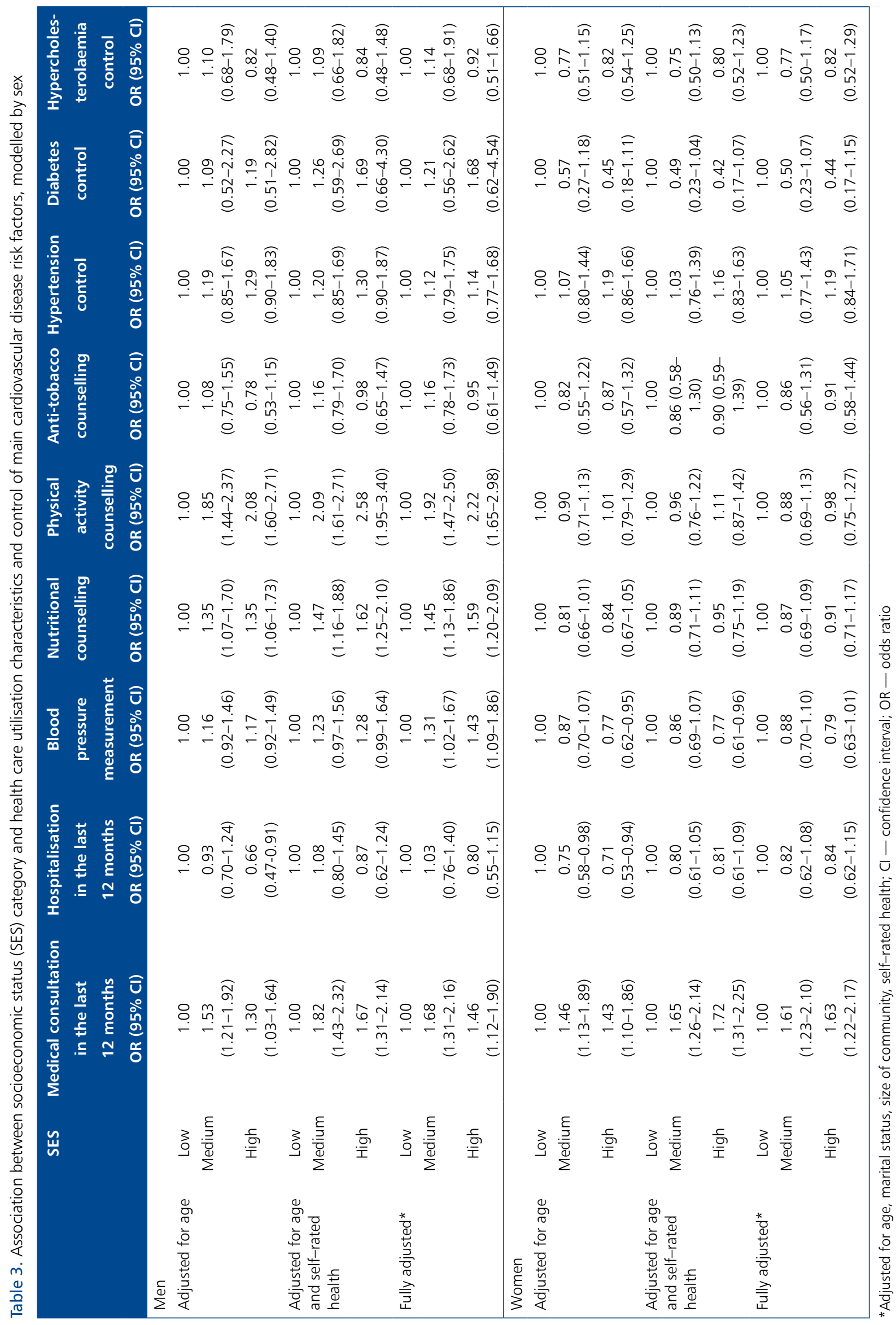


holders were persons with the highest income, while only $1 \%$ of the holders belonged to the lowest income category [16]. More frequent medical visits among people with higher SES may be due to their greater health competence, better knowledge of alarming symptoms, or better ability to use the system. However, evidence from other countries shows that a higher level of health competence is not related with the frequency of use of the health care system or that the association is inverse [17-21]; nor were the higher competences predictive of medical assistance overuse [22]. More frequent medical visits in Poles with high SES may indicate that the better availability of the health care system was related to a better financial situation.

The fact that men are more likely to receive counselling on CVD risk factors is known. Sex differences in benefits from health care were subjected to various assessments, indicating the presence of gender bias in the health systems. For example, the analysis of the Polish health system shows that male patients seem to be in an advantageous position: the mean reimbursement per service for men was higher in most medical care areas, and men reported fewer problems with access to health care [23]. Also, the European Commission Report on sex inequalities in health care access indicated that in Poland obligatory health insurance covers the vast majority of the population, but women are the majority among the few uninsured. In men the level of private health spending is higher and voluntary private insurance coverage is two times higher compared to women. Women more often declare discrimination and unequal treatment in using health services [24]. Several studies indicated that men derive greater benefit from the health care system $[25,26]$. However, the results are not entirely consistent in all studies; some of them reported more beneficial effect of health care in women [27, 28]. Middle-aged men are at higher risk of CVD compared to middle-aged women, so it is reasonable to implement higher intensity of preventive counselling in them, but there is no medical justification for higher frequency of CVD risk factor counselling in higher SES classes. Ostrowska [29] noted that wealthier patients had longer consultations and received more attention and more accurate explanations from the doctor. It cannot be excluded, however, that the study participants with higher SES, feeling more responsible for their health, expected some consultations in terms of a healthy lifestyle, and consequently recalled such consultations better during the interview.

Nevertheless, our results suggest that it is unlikely that the social gradient in CVD mortality observed in the Polish population can be explained by differences in access to health care and consequential differences in risk factors control. In the entire sample the prevalence of the main CVD risk factors (hypertension, current smoking, obesity, and dyslipidaemia) was higher in participants with low SES [6]. It seemed that the CVD risk factors could be more effectively controlled by people with higher SES due to higher health competences. Indeed, in our study, persons with higher SES more often used medical consultations and men with higher SES had more complex consultations in terms of CVD risk factor counselling, but control of the main CVD risk factors did not differ across the SES categories. It seems that persons with higher SES in fact have the same risk resulting from exposure to major risk factors, and possibly more frequent consultations do not lead to risk reduction through successful risk factors control.

There are several limitations in the interpretation of the present results which should be considered. First, the results come from a cross-sectional study in which the issue of causality cannot be resolved. Low SES can be regarded as a barrier in the access to proper medical services, but it is possible that worsening of health can lead to lowering of SES. Second, similarly to numerous other studies, we used education and income to determine SES, while it may be also assessed by other characteristics that were not measured in our study. However, our index comprises two important components that determine SES in the Polish society. Third, the division into three groups, which assumes an equal number of people with high, moderate, and low SES, may not reflect the real distribution of SES categories in the population. However, this approach seems the only reasonable solution because there are no separate traditional social classes in the Polish society and it would be hard to create such a stratification. Social classes began to re-establish during the last three decades, after the transition. Recent studies show that the class structure of the Polish society has been undergoing modernisation in the sense that its pattern has begun to be noticeable and seems close to that prevailing in Western Europe [30]. Fourth, the studied group comprised persons aged $\geq 20$ years, so some of the 254 participants aged less than 25 years could have their SES slightly underestimated if they had not received a university degree before the examination. However, sensitivity analysis performed with the exclusion of participants between 20 and 24 years old demonstrated no substantial differences in the results. Fifth, data on SES were missing in about $15 \%$ of the study population which could have biased the results. Indeed, data on SES were slightly more frequently available in participants from small towns, those with better self-rated health, and those who used medical consultations $3 \%$ more often, but no other characteristic was statistically affected by SES data availability. So, it seems that the effect of missing SES data was not substantial. Sixth, the definition of diabetes control was based on fasting glucose (not on glycated haemoglobin), which is not in line with the current guidelines for clinical practice, but such a proceeding is accepted in epidemiological population studies that require simple and reasonably inexpensive diagnostic methods [31]. Seventh, although the sample was selected to represent the population of Poland, the participation rate in the WOBASZ II study was rather low. Consequently the sample examined might not be 
fully representative, and caution in generalising the results to the whole population is recommended. However, the percentages of medical consultations in the last 12 months in our study are virtually the same as those estimated in 2014 by the Central Statistical Office for Poland [32]. Also, our response rate does not seem to be outstandingly lower than the rates observed in other contemporary population studies [33]. Nonparticipation is estimated to be generally higher in low socioeconomic groups, so it is possible that the associations between SES and health care might be stronger than reported.

Nevertheless, several strengths of the study have to be mentioned, such as the large, nationwide sample, targeted at being representative of the population of Poland. The observation did not cover the specific high-risk group but instead the general population, mostly under the care of GPs. Furthermore, the study strictly adhered to standard research methods to ensure that data quality was equally high in all study centres.

In conclusion, the results obtained support the hypothesis that persons with higher SES have better access to health care in terms of frequency of medical consultations. Men with higher SES more often have their BP measured and receive counselling on nutrition and physical activity. However, more complex consultations in high-SES men are not followed by better CVD risk factors control in this group. This may suggest that the gradient in CVD mortality is not a consequence of differences in health care utilisation across the SES categories.

\section{Acknowledgements}

The authors express special thanks to the entire research team and collaborating persons from the field centres in 16 voivodships and to all participants of the WOBASZ II study.

Conflict of interest: Andrzej Pająk: honoraria from Amgen and Sanofi, not related with the present work.

\section{References}

1. Groenhof JP, Mackenbach AM, Cavelaars AE, et al. Socioeconomic inequalities in cardiovascular disease mortality. An international study. Eur Heart J. 2000; 21(14): 1141-1151.

2. Kim YJ, Lee JS, Park J, et al. Trends in socioeconomic inequalities in five major risk factors for cardiovascular disease in the Korean population: a cross-sectional study using data from the Korea National Health and Nutrition Examination Survey, 2001-2014. BMJ Open. 2017; 7(5): e014070, doi: 10.1136/bmjopen-2016-014070, indexed in Pubmed: 28515188.

3. Mackenbach JP, Stirbu I, Roskam AJR, et al. European Union Working Group on Socioeconomic Inequalities in Health. Socioeconomic inequalities in health in 22 European countries. NEngl J Med. 2008; 358(23): 2468-2481, doi: 10.1056/NEJMsa0707519, indexed in Pubmed: 18525043.

4. Wojtyniak B. Cardiovascular disease as a priority of public health. In: Szymborski J. Strzelecki Z. [Incidence and mortality from cardiovascular disease and demographic situation of Poland] Warsaw: Rządowa Rada Ludnościowa, 2015.

5. Podolecka E, Doryńska A, Nadrowski P, et al. Socioeconomic status and cardiovascular risk SCORE. Kardiol Pol. 2018; 76(3): 560-565, doi: 10.5603/KP.a2017.0253, indexed in Pubmed: 29297197.
6. Nadrowski P, Podolecka E, Pajak A, et al. How does the risk of cardiovascular death and cardiovascular risk factor profiles differ between socioeconomic classes in Poland: A country in transition. Cardiol J. 2018 [Epub ahead of print], doi: 10.5603/CJ.a2018.0003, indexed in Pubmed: 29570212.

7. Wojtyniak B, Goryński P, Moskalewicz B, eds. Health situation of Polish citizens and its determinants. Warsaw: National Institute of Public Health - The National Hygiene Institute, 2012.

8. Health and Health Care in 2013. Statistical Information and Elaborations. Warsaw: Central Statistical Office, 2014.

9. Sørensen K, Pelikan JM, Röthlin F, et al. Health literacy in Europe: comparative results of the European health literacy survey (HLS-EU) Eur J Public Health. 2015; 25(6):1053-1058, doi: 10.1093/eurpub/ckv043, indexed in Pubmed: 25843827.

10. Słońska Z, Borowiec A, Aranowska A. Health literacy and health among the elderly: status and challenges in the context of the Polish population aging process. Anthropol Rev. 2015; 78(3): 297-307, doi: 10.1515/anre-2015-0023.

11. Drygas W, Niklas AA, Piwońska A, et al. Multi-centre National Population Health Examination Survey (WOBASZ II study): assumptions, methods, and implementation. Kardiol Pol. 2016; 74(7): 681-690, doi: 10.5603/KP.a2015.0235, indexed in Pubmed: 26620680

12. Kozakiewicz K, Podolecka E, Kwaśniewska M, et al. Association between socioeconomic status and cardiovascular risk. Kardiol Pol. 2016; 74(2): 179-184, doi: 10.5603/KP.a2015.0139, indexed in Pubmed: 26202533.

13. Panagiotakos DB, Pitsavos CE, Chrysohoou CA, et al. The association between educational status and risk factors related to cardiovascular disease in healthy individuals: The ATTICA study. Ann Epidemiol. 2004; 14(3): 188-194, doi: 10.1016/S10472797(03)00117-0, indexed in Pubmed: 15036222.

14. Pająk A, Szafraniec K, Polak M, et al. Changes in the prevalence, treatment, and control of hypercholesterolemia and other dyslipidemias over 10 years in Poland: the WOBASZ study. Pol Arch Med Wewn. 2016; 126(9): 642-652, doi: 10.20452/pamw.3464, indexed in Pubmed: 27452484.

15. Piepoli MF, Hoes AW, Agewall S, et al. 2016 European Guidelines on cardiovascular disease prevention in clinical practice: The Sixth Joint Task Force of the European Society of Cardiology and Other Societies on Cardiovascular Disease Prevention in Clinical Practice (constituted by representatives of 10 societies and by invited experts)Developed with the special contribution of the European Association for Cardiovascular Prevention \& Rehabilitation (EACPR). Eur Heart J. 2016; 37(29): 2315-2381, doi: 10.1093/eurheartj/ehw106, indexed in Pubmed: 27222591.

16. Central Statistical Office of Poland. [Health and Healthcare in 2015]. Warsaw, 2017.

17. Lee SYD, Tsai TI, Tsai YW, et al. Health literacy, health status, and healthcare utilization of Taiwanese adults: results from a national survey. BMC Public Health. 2010; 10: 614-621, doi: 10.1186/1471-2458-10-614, indexed in Pubmed: 20950479.

18. Rasu RS, Bawa WA, Suminski R, et al. Health Literacy Impact on National Healthcare Utilization and Expenditure. Int J Health Policy Manag. 2015; 4(11): 747-755, doi: 10.15171/ijhpm.2015.151, indexed in Pubmed: 26673335.

19. Karimi S, Keyvanara M, Hosseini M, et al. The relationship between health literacy with health status and healthcare utilization in 18-64 years old people in Isfahan. J Educ Health Promot. 2014; 3: 75, doi: 10.4103/2277-9531.134910, indexed in Pubmed: 25077168.

20. MacLeod S, Musich S, Gulyas S, et al. The impact of inadequate health literacy on patient satisfaction, healthcare utilization, and expenditures among older adults. Geriatr Nurs. 2017; 38(4): 334-341, doi: 10.1016/j.gerinurse.2016.12.003, indexed in Pubmed: 28089217. 
21. van der Gaag M, van der Heide I, Spreeuwenberg PMM, et al. Health literacy and primary health care use of ethnic minorities in the Netherlands. BMC Health Serv Res. 2017; 17(1): 350, doi: 10.1186/s12913-017-2276-2, indexed in Pubmed: 28506230.

22. Mantwill S, Schulz P. Low health literacy and healthcare utilization among immigrants and non-immigrants in Switzerland. Patient Educ Couns. 2017; 100(11): 2020-2027, doi: 10.1016/j. pec.2017.05.023.

23. Łyszczarz B. Gender bias and sex-based differences in health care efficiency in Polish regions. Int J Equity Health. 2017; 16(1): 8, doi: 10.1186/s12939-016-0501-y, indexed in Pubmed: 28077152.

24. European Comission. Access to healthcare and long-term care: Equal for women and men? Luxembourg: Publications Office of the European Union, 2009.

25. Asiskovitch S. Gender and health outcomes: the impact of healthcare systems and their financing on life expectancies of women and men. Soc Sci Med. 2010; 70(6): 886-895, doi: 10.1016/j. socscimed.2009.11.018, indexed in Pubmed: 20092922.

26. Health status determinants: Lifestyle, environment, health care resources and efficiency. Paris: OECD Economics Department Working Papers, 2008: 627.
27. Nixon J, Ulmann P. The relationship between health care expenditure and health outcomes. Eur J Health Econ. 2006; 7(1): 7-18, doi: 10.1007/s10198-005-0336-8.

28. Poças A, Soukiazis E. Health status determinants in the OECD countries. A panel data approach with endogenous regressors. GEMF Working Papers. 2010: 4.

29. Ostrowska A. [Psychosocial determinants of health inequalities]. Zdrowie Publiczne i Zarządzanie. 2011; 2: 55-63.

30. Słomczyński KM, Janicka K, Tomescu-Dubrow I. On the relationship between class structure and social stratification. Warsaw: Zespół Porównawczych Analiz Nierówności Społecznych, Instytut Filozofii i Socjologii Polskiej Akademii Nauk, 2014.

31. Engelgau MM, Thompson TJ, Herman WH, et al. Comparison of fasting and 2-hour glucose and HbA1c levels for diagnosing diabetes. Diagnostic criteria and performance revisited. Diabetes Care. 1997; 20(5): 785-791, indexed in Pubmed: 9135943.

32. Central Statistical Office of Poland. [Health status of population of Poland in 2014]. Warsaw, 2016.

33. Galea S, Tracy M. Participation rates in epidemiologic studies. Ann Epidemiol. 2007; 17(9): 643-653, doi: 10.1016/j.annepidem.2007.03.013, indexed in Pubmed: 17553702.

Cite this article as: Kozela M, Polak M, Doryńska A, et al. Socioeconomic and sex differences in health care utilisation, counselling on cardiovascular disease (CVD) risk factors, and CVD risk factors control in the Polish population. The WOBASZ II Study. Kardiol Pol. 2018; 76(11): 1516-1524, doi: 10.5603/KP.a2018.0176.

\section{WHAT IS NEW?}

Social status is considered as one of the determinants of health status inequities. Indeed, our results from a nationwide population study of a representative sample indicate that persons with high socioeconomic status (SES) more often used medical consultations than persons with low SES, regardless of the sex. Moreover, men with high SES were found to receive more counselling on cardiovascular disease (CVD) risk factors than men with low SES. However, in terms of objective health outcomes SES did not differentiate the Polish population. No significant differences were found in the frequency of hospitalisations or control of main CVD risk factors between different SES subgroups. 